\title{
Reduction of circulating innate lymphoid cell progenitors results in impaired cytokine production by innate lymphoid cells in patients with lupus nephritis
}

Seungwon Ryu', Eun Young Lee ${ }^{2}$, Dong Ki Kim, ${ }^{3,4}$, Yon Su Kim³,4, Doo Hyun Chung ${ }^{5,6}$, Ji Hyung Kim², Hajeong Lee $\mathrm{L}^{3,4^{*+}}$ and Hye Young Kim ${ }^{1,8^{*}+}$ (D)

\begin{abstract}
Background: Innate lymphoid cells (ILCS) play an essential role in maintaining homeostasis; however, they can also cause chronic inflammation and autoimmune disease. This study aimed to identify the role of ILCs in the pathogenesis of lupus nephritis (LN).

Methods: The percentage of ILCs within the peripheral blood mononuclear cell (PBMC) population and urine of patients with LN $(n=16)$, healthy controls $(H C ; n=8)$, and disease controls (ANCA-associated vasculitis (AAV; $n=6)$, IgA nephropathy (IgAN; $n=9)$, and other glomerular diseases $(n=5)$ ) was determined by flow cytometry analysis. In addition, ILCs were sorted and cultured with plasma from LN patients or HC to elucidate whether the reduced population of CD117 ILCs observed in LN was due to changes in the ILC progenitor population.

Results: The percentage of total ILCS and CD $117^{+}$ILCS in LN was significantly lower than that in HC. The percentage of cytokine-secreting ILCs was also lower in LN; however, when the disease stabilized, cytokine production was restored to levels similar to those in HC. The increase in the number of exhausted ILCs (cells unable to secrete cytokines) correlated positively with disease activity. When CD $117^{+} \mathrm{ILCS}$ were cultured with LN plasma, the number of CD117 $7^{+} \mathrm{LCS}$ fell, but that of other ILC subsets increased.

Conclusions: The percentage of CD117 ILCS and the capacity of ILCS to secrete cytokines fell as LN severity increased, suggesting that an inflammatory environment of $L N$ induces persistent differentiation and exhaustion of ILCS.
\end{abstract}

Keywords: Lupus nephritis, Autoimmune kidney disease, Innate lymphoid cells (ILCS), Cytokine

\section{Introduction}

Lupus nephritis (LN) is a renal inflammation caused by systemic lupus erythematosus (SLE). SLE is characterized by manifestations of various organs and the presence of autoantibodies such as anti-double-stranded DNA (antidsDNA) and anti-Smith antibodies [1]. The organ most

\footnotetext{
* Correspondence: mdhjlee@gmail.com; hykim11@snu.ac.kr

${ }^{\dagger}$ Hajeong Lee and Hye Young Kim are senior authors and contributed equally to this work.

${ }^{3}$ Division of Nephrology, Department of Internal Medicine, Seoul National University Hospital, Seoul, South Korea

'Laboratory of Mucosal Immunology, Department of Biomedical Sciences, Seoul National University College of Medicine, Seoul 03080, South Korea Full list of author information is available at the end of the article
}

commonly affected is the kidney. Moreover, invasion of the kidney is a major prognostic factor for long-term morbidity and mortality for patients with SLE [1]. Genetic and environmental factors contribute to the pathogenesis of $\mathrm{LN}$; however, the exact mechanism is unclear. Genomewide association studies of patients or mouse models of lupus nephritis suggest that protection from autoantigens (e.g., nucleic acids or histone proteins) induced immune reactions is compromised at various checkpoints $[2,3]$. Renal inflammation, mainly glomerulonephritis, persists with autoantibodies that cross-react with endogenous glomerular antigens. In addition, deposition of immune complexes within glomeruli recruits immune cells via

(c) The Author(s). 2020 Open Access This article is distributed under the terms of the Creative Commons Attribution 4.0 International License (http://creativecommons.org/licenses/by/4.0/), which permits unrestricted use, distribution, and 
complement activation, thereby aggravating the inflammation [4]. Current treatments for severe LN are based on nonspecific immunosuppression by high-dose glucocorticoids along with cytotoxic agents such as cyclophosphamide or mycophenolate mofetil, all of which have dose-limiting side effects [5]. Thus, the development of novel therapeutic targets based on the specific pathogenesis of the disease is needed [6,7].

Innate lymphoid cells (ILCs) are a newly discovered type of immune cell that is characterized by antigenindependent activation. Although they do not react in an antigen-specific manner, ILCs resemble $\mathrm{T}$ cells in that they share key transcription factors and produce cytokines. Group 1 ILCs (ILC1s) including NK cells express T-bet and produce IFN- $\gamma$ and TNF- $\alpha$. Group 2 ILCs (ILC2s) express GATA3 and produce mainly type 2 cytokines such as IL-5 and IL-13. Group 3 ILCs (ILC3s) express RORC and produce IL-17 and/or IL-22 [8]. Conventionally, human and mouse ILCs are classified according to the expression of surface markers; Lin $^{-}$ (Lineage (Lin) markers; a collection of surface markers of immune cells, not those of ILCs) $\mathrm{CD} 127^{+}$lymphocytes. Among ILC subsets, $\mathrm{Lin}^{-} \mathrm{CD} 127^{+} \mathrm{CD} 117^{+}$lymphocytes are considered as ILC3s; however, the current thinking is that circulating $\mathrm{CD} 117^{+}$ILCs are mainly progenitor cells rather than ILC3s $[9,10]$.

Although ILCs play roles in several autoimmune diseases, including rheumatoid arthritis, juvenile inflammatory arthritis, spondyloarthritis, and systemic sclerosis [11-14], their role in autoimmune kidney diseases is unclear. Recently, ILC2s were identified as a major ILC population in the healthy kidneys of mice [15]. These cells are located in both glomerular and tubulointerstitial areas following the vasculature of a mouse kidney [15, 16]. Work in murine models of renal disease suggests that ILC2s protect the kidney from toxic [15] or ischemic kidney injury $[17,18]$ by producing type 2 cytokines and amphiregulin. In addition, a murine model of LN revealed a reduced percentage of ILC2s in the injured kidney, but in vivo expansion of ILC2s (mediated by IL-33) ameliorated kidney inflammation; this suggests a protective role of ILCs [19]. However, human data supporting these results are scarce. Here, we examined the role of ILCs in LN and other autoimmune kidney diseases by analyzing ILC populations isolated from both peripheral blood and urine samples from LN patients, disease controls, and healthy controls $(\mathrm{HC})$.

\section{Methods}

\section{Subjects}

We enrolled active LN patients and active disease controls when they received diagnostic kidney biopsy in the Seoul National University Hospital, Korea. Stable LN patients were enrolled during their outpatient department visits.
Live kidney donors were included as healthy controls (HC) when they donated their kidneys altruistically. LN patients met the criteria of either the 1997 update of the 1982 American College of Rheumatology Revised Criteria for Classification of Systemic Lupus Erythematosus [20] or the 2012 Systemic Lupus International Collaborating Clinics Classification Criteria for Systemic Lupus Erythematosus [21]. The stable LN was defined as patients without any systematic manifestations of SLE and normal renal function. Disease controls included autoimmune kidney disease controls (anti-neutrophil cytoplasmic antibody (ANCA)-associated vasculitis (AAV) and IgA nephropathy $(\operatorname{IgAN}))$ and other glomerular renal disease controls (minimal change disease (MCD) and focal segmental glomerulosclerosis (FSGS)). We obtained blood, urine, or renal tissues from patients with active $\mathrm{LN}$, disease control, and HCs, whereas only blood samples from those with stable LN. Information on demographic factors, clinical manifestations, and laboratory tests was reviewed at the time of sample acquisition (Table 1).

\section{Cell isolation from peripheral blood, urine, and kidney tissue}

Peripheral blood mononuclear cells (PBMCs) were isolated in a manner of density-gradient separation principles using Ficoll-Paque ${ }^{\mathrm{Tm}}$ PLUS (GE Healthcare, Uppsala, Sweden) as previously described [22]. Urine cells were washed and resuspended in 40\% Percoll $^{\text {Tw }}$ (GE Healthcare, Uppsala, Sweden), which was layered onto $80 \%$ Percoll solution. Leukocytes from urine samples were obtained from the interface between two Percoll layers after centrifugation at $1800 \mathrm{rpm}, 4^{\circ} \mathrm{C}$ for $25 \mathrm{~min}$. A normal portion of nephrectomized kidney from renal cell carcinoma, used for comparing the characteristics of ILCs from kidney and urine, was mechanically and enzymatically dissociated for single-cell preparation as described previously [17].

\section{Flow cytometry and sorting of ILCs}

Single cells from either blood, urine, or renal tissue were blocked with human BD Fc Block ${ }^{\mathrm{Tm}}$ (BD Biosciences, NJ, USA). Cells were stained with fluorescent monoclonal antibodies for $30 \mathrm{~min}$ at $4{ }^{\circ} \mathrm{C}$. For intracellular staining, cells were fixed and permeabilized with BD fixation/ permeabilization solution kit (BD Biosciences, NJ, USA) for $20 \mathrm{~min}$ at $4{ }^{\circ} \mathrm{C}$. Cells investigated for cytokine production were stimulated with PMA $(100 \mathrm{ng} / \mathrm{ml}$; SigmaAldrich, MO, USA)/ionomycin $(1 \mu \mathrm{g} / \mathrm{ml}$; Sigma-Aldrich, $\mathrm{MO}$, USA) and incubated with GolgiStop ${ }^{\mathrm{mm}}(0.7 \mu \mathrm{L} / \mathrm{ml}$; BD Biosciences, NJ, USA) for $4 \mathrm{~h}$ at $37^{\circ} \mathrm{C}$. Cytokines were stained with fluorescent monoclonal antibodies overnight at $4{ }^{\circ} \mathrm{C}$. Antibodies used for flow cytometric analysis or cell sorting were as follows: lineage markers (anti-human CD3 (clone: UCHT1), anti-human CD19 (HIB19), anti-human FceRI $\alpha$ (AER-37), anti-human 
Table 1 Characteristics of the study group

\begin{tabular}{|c|c|c|c|c|c|c|}
\hline & $\begin{array}{l}\text { Active } \operatorname{LN}(n= \\
16)\end{array}$ & $\begin{array}{l}\text { Stable LN }(n= \\
\text { 10) }\end{array}$ & $\operatorname{AAV}(n=6)$ & $\operatorname{lgAN}(n=9)$ & $\begin{array}{l}\text { MCD/FSGS (MCD, } n=4 ; \text { FSGS, } n= \\
\text { 1) }\end{array}$ & $\mathrm{HC}(n=8)$ \\
\hline Age (years) & $31(23-45)$ & $53(31.75-61.25)$ & $52(45.5-59.5)$ & $45(35.5-57)$ & $53(40.5-62)$ & $\begin{array}{l}49.5(40.5- \\
55.5)\end{array}$ \\
\hline Female, $n(\%)$ & 15 (93.75) & $10(100)$ & $4(66.67)$ & 8 (88.89) & $3(60)$ & $6(75)$ \\
\hline WBC count, $\times 10^{3} / \mathrm{mL}$ & $4.43(3.32-6.54)$ & $4.30(3.67-4.89)$ & $9.98(6.89-15.37)$ & $8.1(7.36-8.35)$ & $8.02(6.28-10.01)$ & $\begin{array}{l}5.44(4.56- \\
7.77)\end{array}$ \\
\hline CRP, mg/L & $0.15(0.07-1.15)$ & $0.05(0.03-0.16)$ & $2.21(0.38-10.35)$ & $\begin{array}{l}0.03(0.01- \\
0.15)\end{array}$ & $0.04(0.02-0.05)$ & $\begin{array}{l}0.02(0.01- \\
0.05)\end{array}$ \\
\hline Serum BUN, mg/dL & $14.5(12-18.75)$ & $\begin{array}{l}15.5(12.25- \\
22.25)\end{array}$ & $31(21.75-58.75)$ & $17(14.5-27)$ & $14(11.5-23.5)$ & $15(11-16)$ \\
\hline UPCR, mg/mg & $2.93(0.90-5.87)$ & $0.21(0.09-0.36)$ & $2.16(1.233-2.97)$ & $1.6(0.90-4.62)$ & 9.55 (3.99-16.88) & $\begin{array}{l}0.05(0.04- \\
0.11)\end{array}$ \\
\hline $\begin{array}{l}\text { Serum anti-dsDNA, IU/ } \\
\mathrm{ml}\end{array}$ & $\begin{array}{l}512(267.9- \\
849.8)\end{array}$ & $6.7(2-155.9)$ & N/A & N/A & N/A & N/A \\
\hline $\mathrm{C} 3, \mathrm{mg} / \mathrm{dL}$ & 44.5 (39.5-59.75) & $\begin{array}{l}69.5(47.75- \\
84.75)\end{array}$ & $\begin{array}{l}106.5(93.75- \\
112.3)\end{array}$ & $99(85-124)$ & N/A & N/A \\
\hline $\mathrm{C} 4, \mathrm{mg} / \mathrm{dL}$ & $8(5.5-16)$ & $10.5(4.5-21)$ & $25(12.5-30.25)$ & $28(20.5-32.5)$ & N/A & N/A \\
\hline
\end{tabular}

The data are presented as the median and interquartile range (IQR) or as number (percentage)

Abbreviations: $L N$ lupus nephritis, $A A V$ ANCA-associated vasculitis, IgAN IgA nephropathy, $M C D$ minimal change disease, FSGS focal segmental glomerulosclerosis, $H C$ healthy control, WBC white blood cell, CRP C-reactive protein, BUN blood urea nitrogen, UPCR urine protein to creatinine ratio, dsDNA double-stranded DNA, C3 complement component 3, C4 complement component 4

CD49b (P1E6-C5), anti-human CD11b (ICRF44), antihuman CD11c (3.9), and anti-human CD14 (HCD14)), anti-human CRTH2 (BM16), anti-human CD117 (104D2), anti-human NKp44 (P44-8), anti-human PD-1 (EH12.2H7), anti-human IL-13 (JES10-5A2), anti-human IL-17A (BL168), anti-Annexin V and Streptavidin from Biolegend (CA, USA), and anti-human IFN- $\gamma$ (B27) from $\mathrm{BD}$ Biosciences (NJ, USA). Flow cytometric data were collected by LSRFortessa X-20 (BD Biosciences, NJ, USA) and analyzed by FlowJo v10 (BD Biosciences, NJ, USA). Fluorescence-labeled ILCs were sorted with Aria III (BD Biosciences, NJ, USA).

\section{Immunofluorescence of kidney tissue sections}

Kidney tissues were obtained from percutaneous needle biopsy in cold PBS. Tissues were mounted on Surgipath FSC22 Frozen Section Compound (Leica, IL, USA) and stored at $-80{ }^{\circ} \mathrm{C}$ until the section of the tissues. As the first step for staining, tissues were fixed with cold acetone. After blocking, primary and secondary antibodies were applied to tissue section and incubated for an hour at room temperature, respectively. Coverslip was mounted on the tissue slides after application of ProLong $^{\mathrm{Tx}}$ Diamond Antifade Mountant with DAPI (Life Technologies, OR, USA) applied to the tissue slides. The antibodies used for the staining were as follows: anti-cKit antibody (host: rabbit, polyclonal) from Biorbyt (Cambridge, UK), anti-CD3 antibody (rat, polyclonal) from Abcam (Cambridge, UK), and anti-rabbit AF594 (donkey, polyclonal) and anti-rat AF488 (donkey, polyclonal) from ThermoFisher Scientific (IL, USA). Slides were imaged by confocal microscopy, FV3000 (Olympus, Tokyo, Japan) and analyzed with FV10-ASW 4.0 Viewer (Olympus, Tokyo, Japan).

\section{Culture of circulating CD117 ${ }^{+}$ILCs}

For whole blood immune cell cultures, $2 \times 10^{6}$ PBMCs were cultured in plasma from healthy controls or $\mathrm{LN}$ with recombinant human IL-2 and IL-7 (40 ng/ml each; R\&D Systems, MN, USA) for $24 \mathrm{~h}$. To test the role of $\mathrm{CD} 117^{+}$ILCs as ILC progenitors in LN condition, CD $117^{+}$ILCs were sorted, and 200-300 cells were cultured with plasma from healthy controls or LN for 8 days. $2.5 \times 10^{3}$ OP9 cells (ATCC, VA, USA) were seeded on a 96-well round-bottom plate a day before CD117 ILC culture, as previously described [9]. Recombinant human IL-2 and IL-7 were added on each well every 3 days. To verify the contribution of IL-1 receptor signaling in the differentiation of blood ILC progenitors, human IL-1R1 blocking antibody $(2 \mu \mathrm{g} / \mathrm{ml}$; R\&D Systems, $\mathrm{MN}$, USA) was added to the whole blood immune cell cultures with HC or LN plasma as the whole blood immune cell culture assay above. Serum level of human IL$1 \beta$ was quantified by ELISA (R\&D Systems, MN, USA).

\section{Statistical analysis}

The percentages of ILCs were compared between various renal disease groups or different disease activity. Two unpaired-groups were compared using Student's $t$ test for the parametric measure or Mann-Whitney test for nonparametric measures. Paired Student's $t$ tests were used to calculate the differential effect on blocking 
IL-1 receptors on blood ILCs. For correlation analysis, the Pearson correlation for a parametric measure or Spearman correlation for a nonparametric measure was used. The determination of the normality of data was based on the Shapiro-Wilk test. Graphs were displayed with median, and the first and third quartiles (Q1-Q3). The significance level was 0.05 for two-tailed tests. Statistical analysis was performed with GraphPad Prism 7 (GraphPad, CA, USA).

\section{Results}

The percentage of CD117 ${ }^{+}$ILCs in blood and urine from patients with LN is lower than that in controls

To determine the distribution of ILCs in patients with $\mathrm{LN}$, we recruited 16 patients with $\mathrm{LN}, 20$ with other renal diseases, and eight $\mathrm{HC}$ (Table 1). Blood ILCs were identified as $\mathrm{CD} 45^{+} \mathrm{Lin}^{-} \mathrm{CD} 127^{+}$lymphocytes. These ILCs were further subdivided according to the expression of surface markers; CRTH2 for ILC2s, CD117 for ILC3s and/or progenitor of ILCs, and CRTH2 $2^{-} / \mathrm{CD} 117^{-}$ for ILC1s (Fig. 1a, b). The percentage of total ILCs within the $\mathrm{CD} 45^{+}$cell population from patients with LN was significantly lower than that in $\mathrm{HC}(P=0.0481)$ (Fig. 1c). We noted that $C D 117^{+}$ILCs in most patients with autoimmune kidney diseases showed reduced numbers (LN $(P=0.0003)$, AAV $(P=0.0877)$, and $\operatorname{IgAN}(P=$ $0.0014)$ ), but not in MCD or FSGS. By contrast, the percentage of $\mathrm{CD}_{117^{-}} / \mathrm{CRTH} 2^{-}$ILC1s in patients with autoimmune kidney diseases (LN, AAV, and IgAN) except MCD/FSGS was higher than that in HC.

Although we observed changes in the proportions of ILCs in the blood of patients with autoimmune kidney diseases including LN, they could not reflect local or kidney-specific immune responses completely. Therefore, we obtained urine samples from patients with LN, AAV, IgAN, and MCD/FSGS and analyzed ILCs by flow cytometry (Fig. 1d, e). ILCs were barely detectable in samples from $\mathrm{HC}$ because of few cell numbers in normal urine. However, the percentage of CD $117^{+}$ILCs in urine from LN patients was significantly lower than that in samples from patients with other renal diseases (LN vs. IgAN $(P=0.0034)$ and LN vs. MCD/FSGS $(P=$ 0.0087)) (Fig. 1e). Also, we confirmed the presence of ILCs in a nephrectomized kidney by flow cytometry (Additional file 1: Figure S1) and examined CD117 ${ }^{+}$ ILCs in renal tissue from $\mathrm{HC}$ and $\mathrm{LN}$ by immunofluorescence (IF) staining (Additional file 2: Figure S2). IF staining revealed that renal tissue from LN harbored fewer $\mathrm{CD} 117^{+}$ILCs than that from healthy control, although the $\mathrm{CD}^{+} \mathrm{T}$ cell count in $\mathrm{LN}$ was higher (Additional file 2: Figure S2d). It implied that not only circulating but also intra-renal CD117 ${ }^{+}$ILCs decreased in patients with $\mathrm{LN}$.

\section{ILCs from patients with LN show reduced cytokine- producing capacity}

To better understand the pathophysiologic function of ILCs in LN, the percentage of IFN- $\gamma-$, IL-13-, and IL17A-producing circulating ILCs was compared with other kidney diseases (AAV, IgAN, and MCD/FSGS), and $\mathrm{HC}$ by flow cytometry (Fig. 2a-c). Blood ILCs from patients with LN produced less cytokines, especially IL13, than ILCs from HC (Fig. 2b, c). The percentage of cytokine-negative ILCs, which do not produce IFN- $\gamma$, IL-13, or IL-17A, was higher in those with autoimmune kidney diseases, including LN, than in HC (Fig. 2e). ILCs from LN patients also showed higher expression of PD-1 than those from $\mathrm{HC}$, suggesting that those cytokine-negative ILCs were "exhausted ILCs" (Fig. 2d). Especially, the PD-1-producing ILCs showed the characteristics of cytokine-negative ILCs (Additional file 5: Figure S5).

We noted that circulating $\mathrm{CD} 117^{+}$ILCs, in patients with LN (Fig. 1c), were significantly lower than that in $\mathrm{HC}$; however, there was no difference in the percentage of IL-17-producing ILCs (which are conventionally regarded as IL-17A $\mathrm{A}^{+}$ILC3s) between $\mathrm{HC}$ and patients with renal disease, including LN (HC vs. LN, $P=0.1405$ ). Instead, IFN- $\gamma$ - and IL-13-producing ILCs (ILC1s and ILC2s, respectively) from patients with $\mathrm{LN}$ and other autoimmune kidney diseases were lower than in $\mathrm{HC}$ (Fig. 2b). Taken together, these data show that the LN microenvironment induces dysfunction of ILCs showing increased expression of PD-1.

\section{The percentage of $\mathrm{CD} 117^{+}$ILCs and cytokine-producing ILCs in LN patients with stable state is higher than in those with active state}

To better understand the implications of the altered percentages of $\mathrm{CD}_{117^{+}}$ILCs in those with $\mathrm{LN}$, we compared the numbers of circulating ILCs in LN patients with the stable state with those in patients with the active state. We found significant differences in ESR (erythrocyte sedimentation rate), UPCR (urine protein to creatinine ratio), and anti-dsDNA levels between the two groups (Fig. 3a). Patients with stable LN had higher numbers of $\mathrm{CD}_{117^{+}}$ILCs and $\mathrm{CRTH}_{2}{ }^{+}$ILCs than those with active LN (Fig. 3b). Consistent with this, we found that the numbers of IL-13- and IL-17A-producing ILCs were higher in those with stable LN (Fig. 3c, d). Finally, patients with stable LN had fewer exhausted ILCs, which expressed higher levels of PD-1 and failed to produce cytokines (Fig. 3c, d). These results imply that the reduced percentage of dysfunctional exhausted ILCs seen in those with active $\mathrm{LN}$ recovers as the disease becomes stable. 


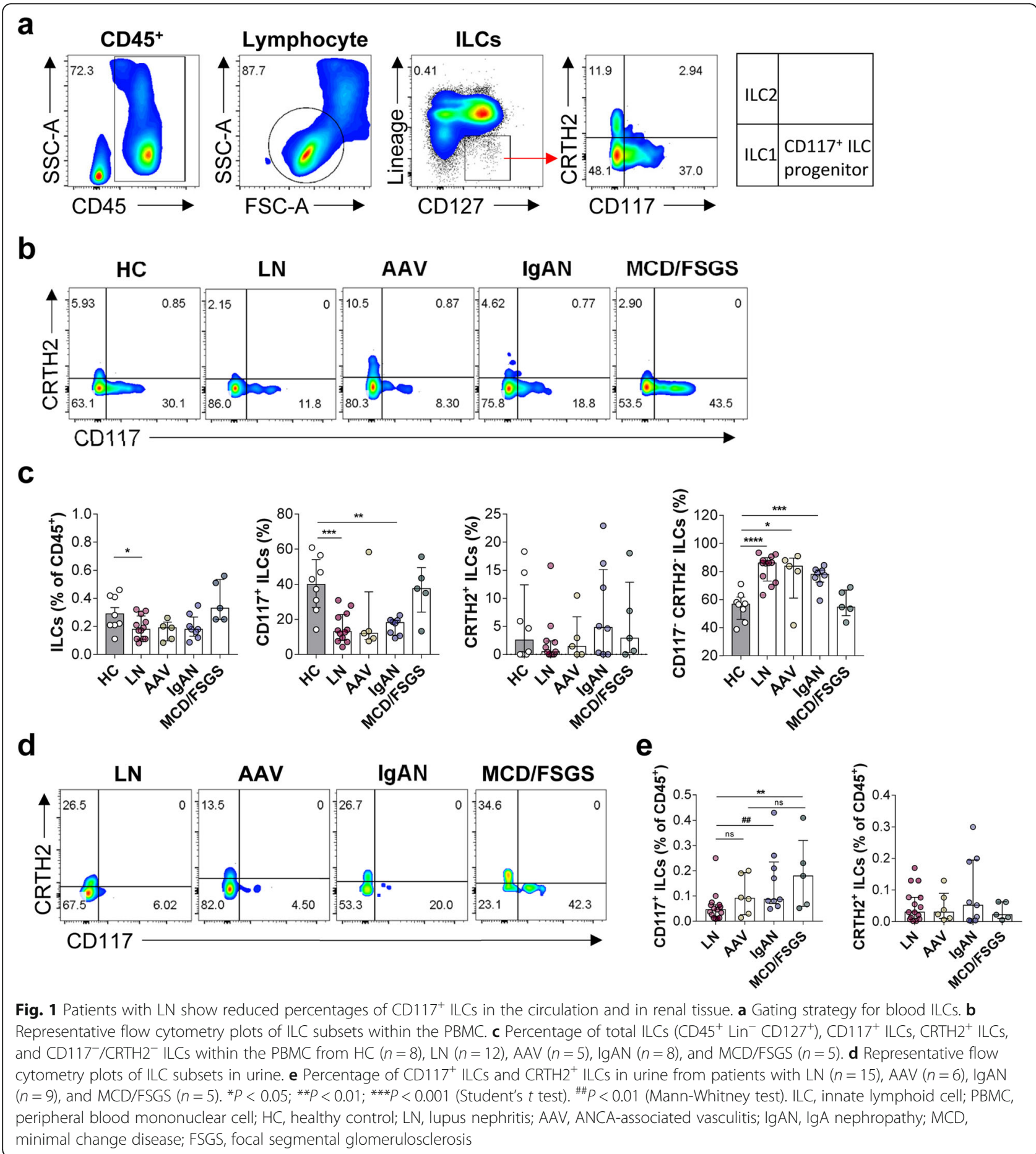

\section{A reduced percentage of $\mathrm{CD} 117^{+} \mathrm{ILC}$ progenitors and functional ILCs reflects disease activity}

Next, we examined the correlation between the total number of ILCs (Fig. 4a), CD $117^{+}$ILC progenitors (Fig. 4b), cytokine-negative ILCs (Fig. 4c), and clinical parameters that reflect disease activity. Here, we found a positive correlation between $\mathrm{CD} 117^{+}$ILC progenitors and white blood cells (WBC; $r=0.5128, P=0.0038$ ), indicating a reduced number of ILCs in cases of active LN accompanying with leukopenia [23]. CD117 ${ }^{+}$ILCs showed a negative correlation with ESR $(r=-0.3968$, $P=0.0675)$ and UPCR $(r=-0.3668, P=0.0503)$. In addition, the percentage of cytokine-negative ILCs correlated positively with clinical indicators of kidney function (UPCR; $r=0.4104, P=0.0373$ ). We also observed a significant reduction in the numbers of CD $117^{+}$ILCs in 


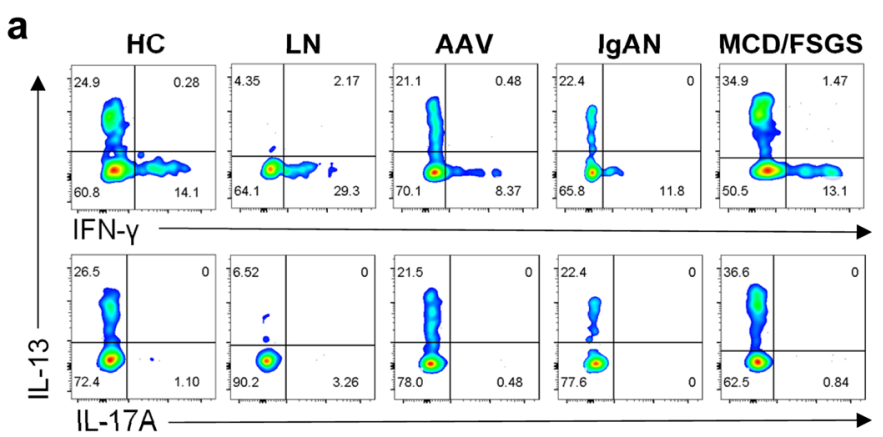

b
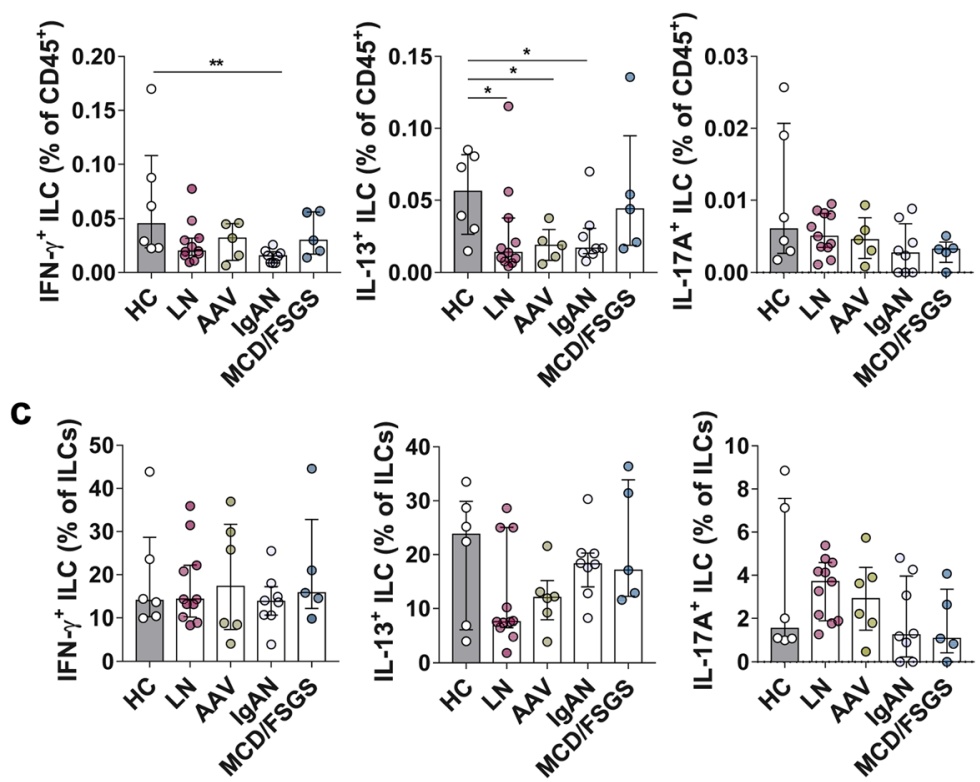

d

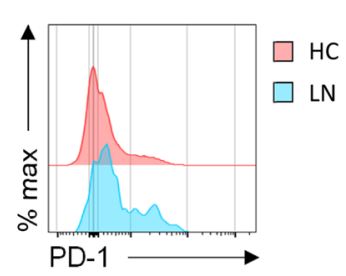

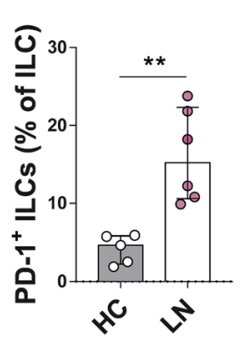

e

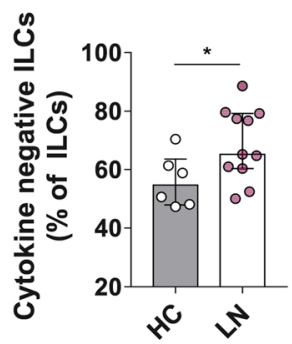

Fig. 2 Circulating ILCS in patients with LN are dysfunctional with respect to cytokine production. a Representative flow cytometry plots of IFN- $\gamma-$, IL-13-, and IL-17A $A^{+}$-producing ILCs within the PBMC. b, c Percentage of IFN- - -, IL-13-, and IL-17A -producing ILCs in (b) CD45 immune cells and (c) total ILCs from HC $(n=6)$, LN $(n=11)$, AAV $(n=5)$, IgAN $(n=8)$, and MCD/FSGS $(n=5)$. d Representative histogram plot of PD-1 expression of circulating ILCs from HC and LN, and percentage of PD- $1^{+} \mathrm{ILCS}$ within PBMC compared between HC $(n=5)$ and LN $(n=6)$. e Percentage of cytokine-negative ILCs within PBMC from HC $(n=6)$ and LN $(n=11)$. ${ }^{*}<<0.05 ;{ }^{*} P<0.01$ (Student's $t$ test)

urine as LN disease activity increased (activity index; based on glomerular and tubulointerstitial changes of LN $(r=-0.6368, P=0.0260)$ ) (Additional file 3: Figure S3a). Taken together, these data suggest that reduced numbers of ILCs in blood and urine could be the possible biomarker of LN severity.
The percentage of $\mathrm{CD} 117^{+}$ILC progenitors in the $\mathrm{LN}$ microenvironment is reduced through increased differentiation

Recent studies show that circulating CD $117^{+}$ILCs might be ILC progenitors rather than ILC3 cells [9, 24]. This, combined with observed patterns in the numbers of IL-17A $\mathrm{A}^{+}$ 
a

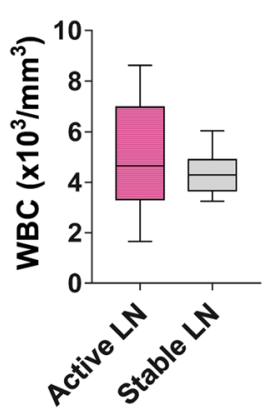

b

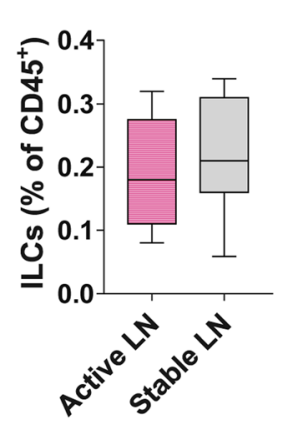

C
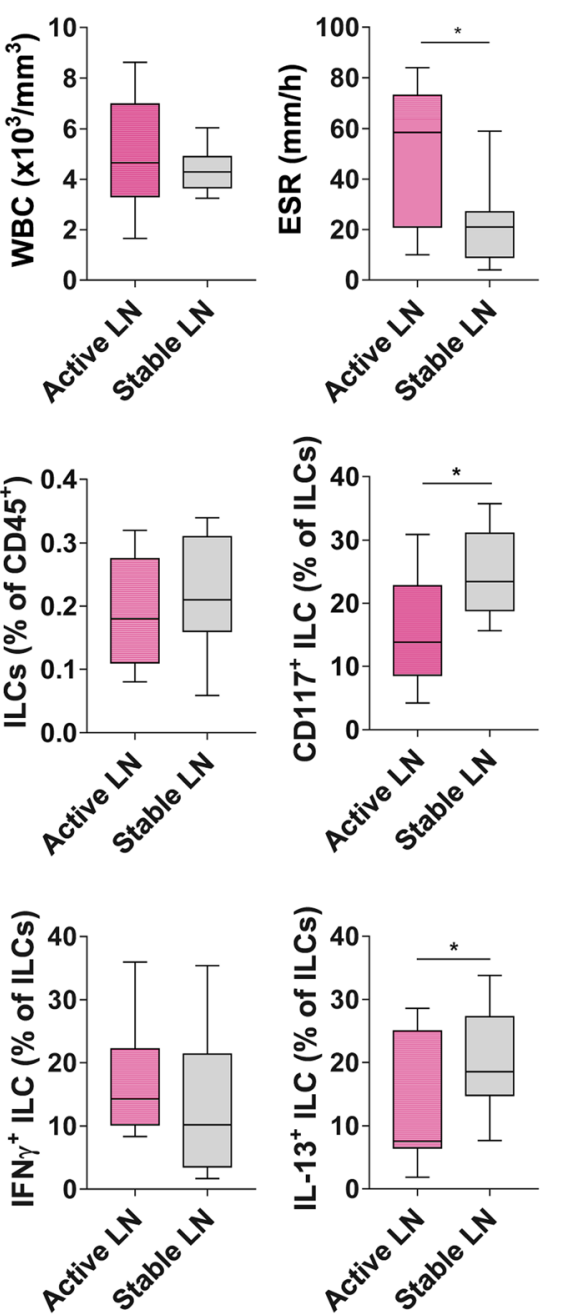
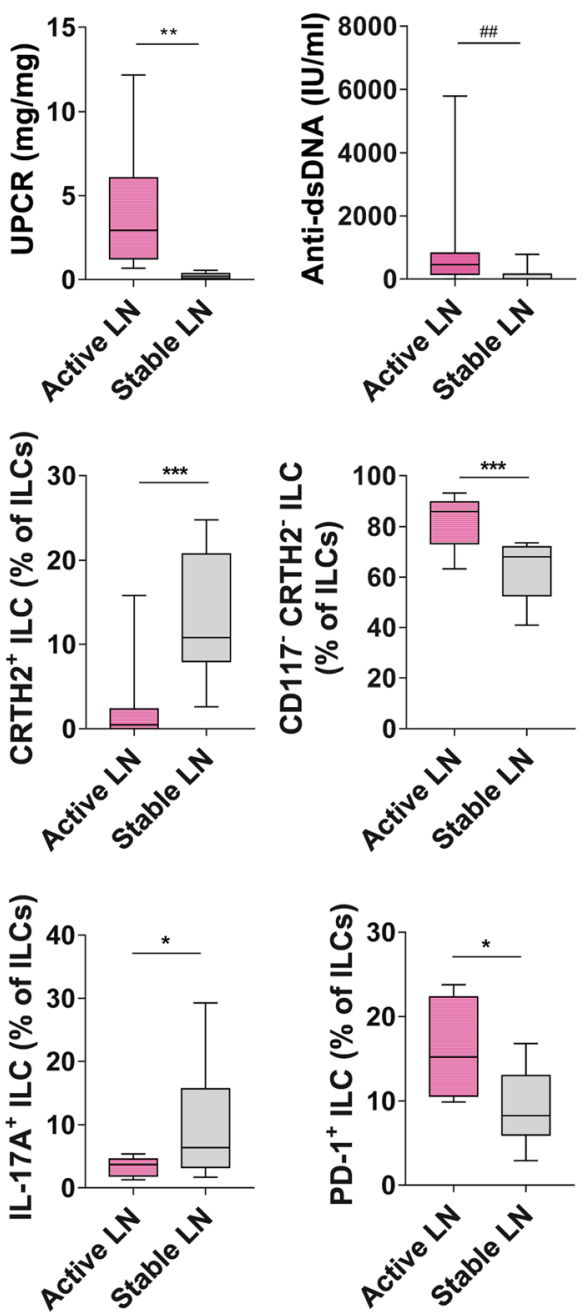

d

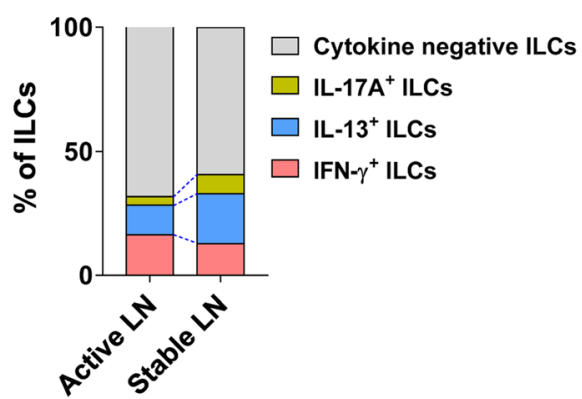

Fig. 3 Patients with stable LN have higher numbers of circulating CD117 $7^{+} \mathrm{LC}$ s and cytokine-producing ILCs. a Differences in clinical parameters

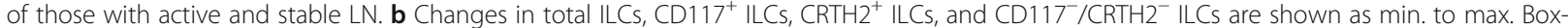
and-whisker plots (line represents the median). $\mathbf{c}$, $\mathbf{d}$ Changes in IFN- $\gamma^{+}, \mathrm{IL}-13^{+}, \mathrm{IL}-17 \mathrm{~A}^{+}, \mathrm{PD}-1^{+} \mathrm{ILCs}$, and cytokine-negative ILCs in PBMC from those with active LN $(n=12)$ or stable LN $(n=10)$. ${ }^{*} P<0.05 ;{ }^{* *} P<0.01 ;{ }^{* * *} P<0.001 ;{ }^{* * * *} P<0.0001$ (Student's $t$ test). ${ }^{\# \#} P<0.01$ (Mann-Whitney test)

ILCs and CD $117^{+}$ILCs in LN, led us to assume that the reduced percentage of $\mathrm{CD} 117^{+}$ILCs may be due to differentiation into other subsets. To verify this, we cultured PBMCs from HC (pre-cultured blood ILCs shown in Additional file 4: Figure S4) with plasma from LN patients to mimic the LN microenvironment in vitro (Fig. 5a). The percentage of CD117 ${ }^{+}$ILC progenitors fell even after culture with healthy plasma; however, this fall was more pronounced after culture with LN plasma (Fig. 5b). By contrast, the percentage of $\mathrm{CD}_{117^{-}} \mathrm{CRTH}^{-}$ILCs 


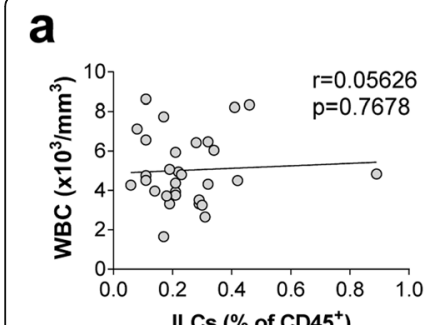

b

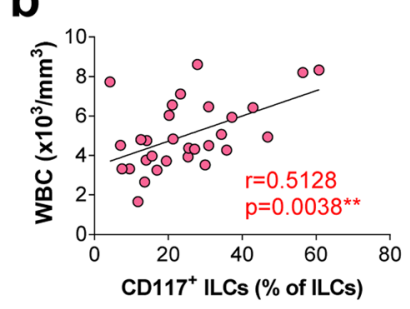

C

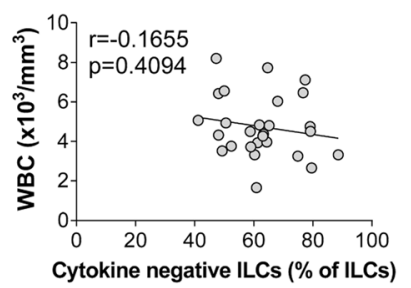

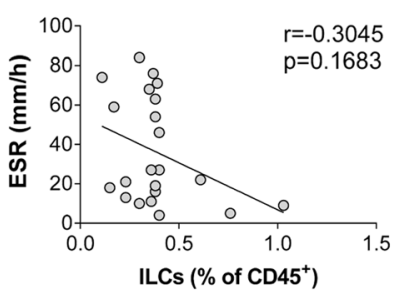
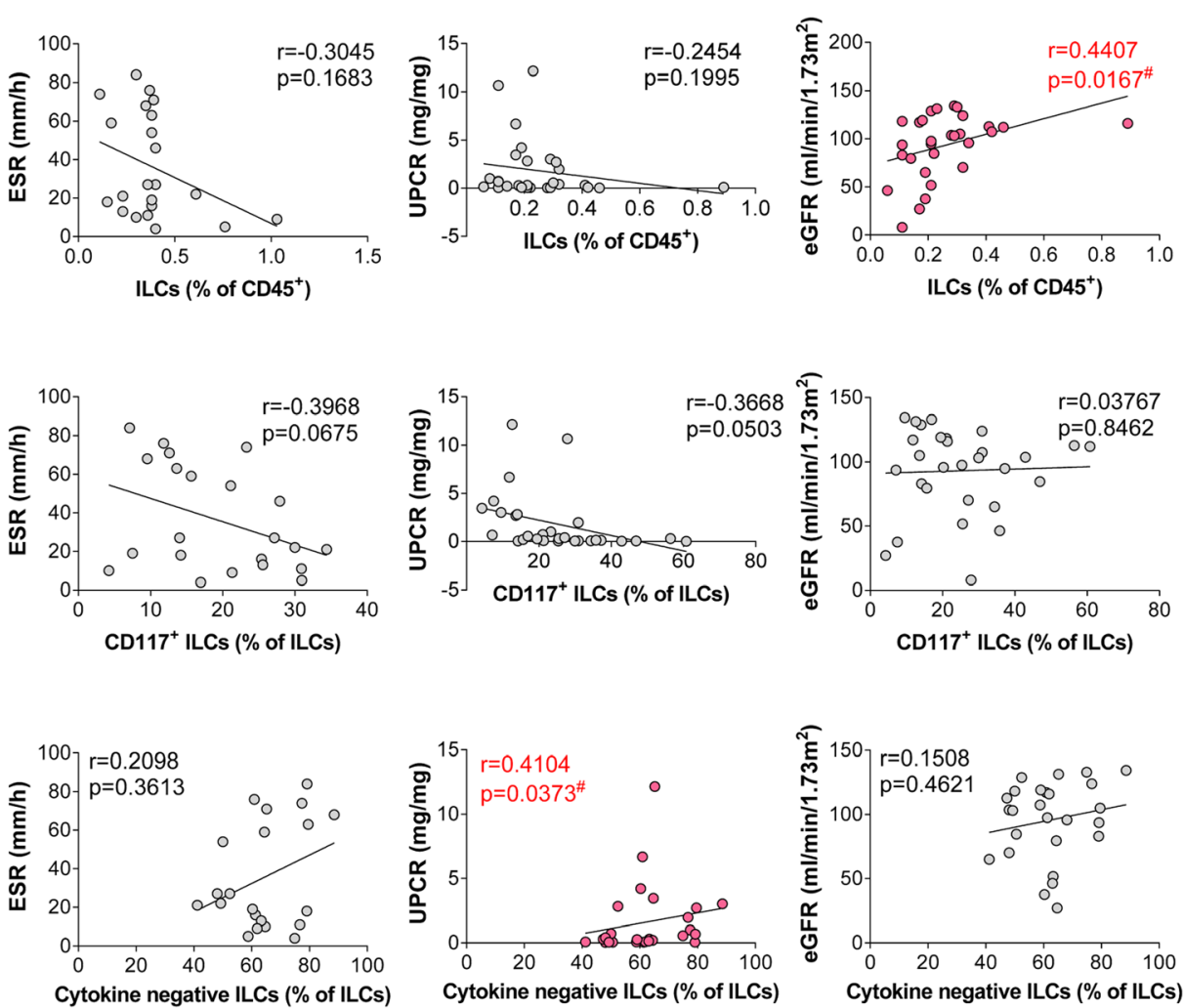

Fig. 4 Circulating ILCs correlate with clinical parameters that reflect LN disease activity. Correlation between the percentage of (a) total ILCs, (b) CD $117^{+} \mathrm{ILCS}$, and (c) cytokine-negative ILCs within PBMC, and clinical parameters (WBC counts, UPCR, and eGFR). **P $<0.01$ (Pearson correlation). ${ }^{\#} P<0.01$ (Spearman correlation). WBC, white blood cell; UPCR, urine protein to creatinine ratio; eGFR, estimated glomerular filtration rate

increased when PBMCs were cultured with plasma from patients with LN (Fig. 5b). However, there is a possibility that the decrease in $\mathrm{CD} 117^{+}$ILCs is due to increased cell death in the LN environment. When we stained ILCs with Annexin V, the apoptosis marker (Fig. 5c, d), cell death was not increased in CD $117^{+}$ILCs or CD $117^{-}$ILCs. Therefore, it excludes the possibilities that cell death caused the reduction of $\mathrm{CD}_{117^{+}}$ILCs, which also implied the progenitorlike characteristics of $\mathrm{CD} 117^{+}$ILCs. To further clarify the hypothesis that increased differentiation of $\mathrm{CD} 117^{+}$ILC progenitors is triggered by soluble factors in LN plasma, we isolated $\mathrm{CD} 117^{+}$ILC progenitors from healthy blood and cultured them with OP9 stromal cells (Fig. 5e). The percentage of $\mathrm{CD}_{117^{+}}$ILC progenitors fell, but that of other ILC subsets increased when exposed to LN plasma (Fig. 5f).

Next, we tried to figure out the soluble factor that contributed to the differentiation of ILC progenitors in LN. IL-1R1 (IL-1 receptor, type 1) exclusively expressed on human blood CD $117^{+}$ILCs than other blood ILC subsets [9]. The recent study of Smith et al. [25] showed that plasma IL-1 $\beta$ highly correlated with renal activity in SLE patients and we also found that serum IL-1 $\beta$ was elevated in LN compared to HC (Fig. 5h). In that sense, we hypothesized that higher IL-1 $\beta$ in active LN plasma would exert the differentiation of $\mathrm{CD} 117^{+}$ILCs through the IL-1R1 signaling pathway. To verify the hypothesis, we used IL$1 \mathrm{R}$ blocking antibody in the PBMC culture assay and compared the change of CD117 ${ }^{+}$ILCs and other ILC subsets (Fig. 5g). Blocking of IL-1 receptor did reverse the changes of CD $117^{+}$ILCs only in LN plasma (Fig. $5 \mathrm{~g}$ ).

Collectively, these data suggest that circulating $\mathrm{CD} 117^{+}$ILCs might be ILC progenitors capable of differentiating into other ILC subsets and that the reduction in the percentage of CD $117^{+}$ILCs in LN might be due to increased differentiation rather than cell death. Elevated IL-1 $\beta$ in LN might be one of the contributors to the differentiation of ILC progenitors in LN.

\section{Discussion}

LN is the kidney manifestation of SLE and a major contributor to lupus-related mortality. The mechanism underlying disease initiation and progression is unclear; therefore, few treatments are available. Here, we characterized ILCs in patients with LN and found that they harbored fewer $\mathrm{CD} 117^{+}$ILCs. Although CD117 ${ }^{+}$ILCs are thought to be ILC3 cells [26], recent findings suggested that $\mathrm{CD} 117^{+}$ILCs are in fact ILC progenitors that can differentiate into any subset of ILCs [9, 27]. We 


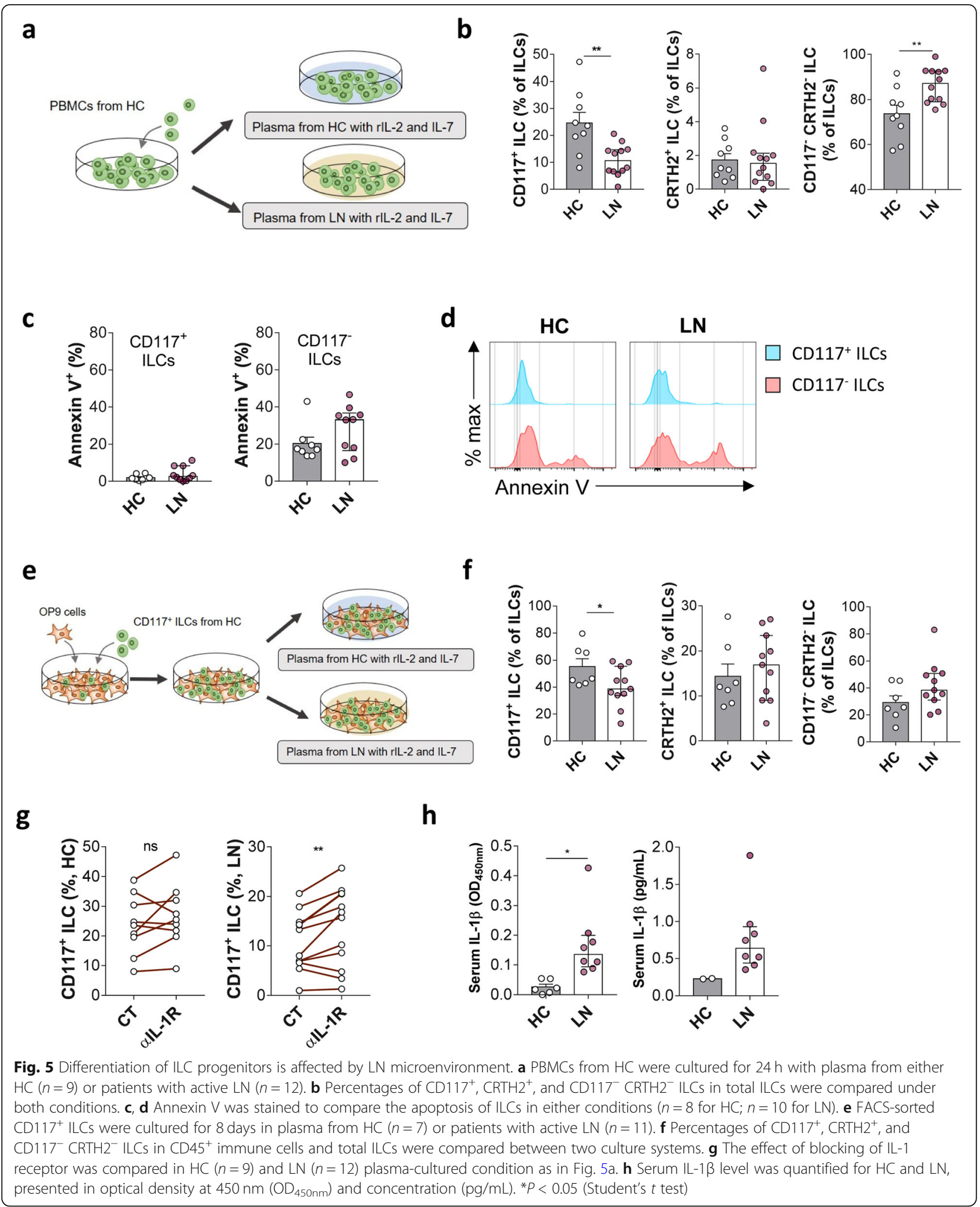

confirmed that this is the case by exposing CD $117^{+}$ILCs to plasma from $\mathrm{HC}$ or $\mathrm{LN}$ patients. Therefore, we assume that changes in the numbers of each subset gave rise to the observed dynamics within the ILC population. The reduced percentage of ILC progenitors and functional ILCs eventually made the ILCs less functional, 
although the underlying mechanism requires further studies (Additional file 6: Figure S6).

Two recent studies reported changes in blood ILC populations in patients with SLE, rather than LN. With an increase in the total number of ILCs in SLE blood, Guo et al. showed that the percentage of ILC1s in peripheral blood from patients with SLE increased, but the percentage of ILC2s and ILC3s decreased [28]. Also, Kruize et al. showed that SLE patients with a high IFN signature had a higher number of ILC1s and a lower number of ILC2s than those with a low IFN signature [29]. Here, we confirmed an increase in the ILC1 (CD117 ${ }^{-} / \mathrm{CRTH}^{-}$ILCs) population in those with $\mathrm{LN}$; however, we did not observe increased IFN- $\gamma$ production. Likewise, there were no changes in the number of ILC2s (CRTH2 ${ }^{+}$ILCs), although the numbers of IL-13producing ILCs were lower in patients with LN than in $\mathrm{HC}$ and the numbers of ILC2s were higher in stable LN compared to active LN. Rather than examining changes in a particular ILC subset based on expression of conventional surface markers, we explored the reduction in ILC numbers in the context of changes in CD $117^{+}$ILC progenitors. The reduction of ILCs was also found in the kidney from lupus mouse [19].

A reduced $\mathrm{CD} 117^{+}$ILC population is not unique to $\mathrm{LN}$; indeed, similar observations were reported for patients infected with HIV or humanized mice infected with simian immunodeficiency virus [30-33]. HIV or simian immunodeficiency virus infection is associated with reduced $\mathrm{CD} 117^{+}$ILC numbers in peripheral blood, tonsil, mesenteric lymph nodes, and colon [30-33]. The decrease in CD $117^{+}$ILCs in those infected with HIV might be due to an overall increase in cell death [31-33]; however, the evidence of cell death was not clear [30]. Kloverpris et al. showed that the number of all ILCs including CD117 ILCs were decreased in HIV infection [30]. If the reduction in ILC numbers in those with HIV infection is not due to an increase in apoptosis, it is plausible that the reduced number of CD $117^{+}$ILC progenitors may result in the reduction in total ILC numbers, as shown in our study. From this perspective, Lim et al. transferred human

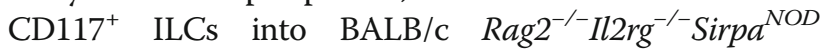
(BRGS) mice that are permissive for robust multi-lineage human hematopoietic stem cell engraftment; they found that CD $117^{+}$ILCs successfully reconstituted ILCs in various organs (lung, gut, liver, and spleen) [9]. A recent study by Nagasawa et al. succeeded in verifying the presence of $\mathrm{CD}_{117^{+}}$ILC progenitors in peripheral organs (nasal polyps) which correspond to the circulating progenitors, but with limited potential for multi-lineage differentiation [34]. These results imply that circulating $\mathrm{CD} 117^{+}$multipotent ILC progenitors can differentiate into tissueresident ILCs to maintain tissue homeostasis. In this study, the blood $\mathrm{CD} 117^{+}$ILCs were examined in vitro whether they could differentiate into the other subsets of ILCs in LN plasma compared to HC plasma. The differentiation of $\mathrm{CD} 117^{+}$ILCs was observed when they were co-cultured with LN plasma.

We also assessed a few other possibilities of reduced CD $117^{+}$ILCs in LN, other than the differentiation of $\mathrm{CD} 117^{+}$ILCs. First, there is a possibility that the decrease in CD117 ${ }^{+}$ILCs is due to increased cell death in the LN environment. The idea is similar to the previous study that IL-17A-producing CD117 ${ }^{+}$ILC3s were reduced in HIVinfected patients [31]. Zhang et al. showed that increased type 1 interferon induced apoptosis of ILC3s. Considering the level of type 1 interferon increased in SLE [35], a decrease of $\mathrm{CD} 117^{+}$ILCs may be due to increased apoptosis. To test this, we analyzed the apoptosis of ILCs and found that $\mathrm{CD} 117^{+}$ILCs were not stained with Annexin V. Therefore, it is hard to say the decrease of CD117 ${ }^{+}$ILCs is due to the increased cell death. Second, the proliferation capacity of CD $117^{+}$ILCs may be lower than that of other subsets of ILCs. When we compared the proliferative activity of CD $117^{+}$ILCs and CD117 ${ }^{-}$ILCs from HC or LN plasmacultured condition, there was no significant difference between HC and LN (data not shown). There is, however, a limitation that short-term culture condition might be unsuitable for detecting proliferation. The last hypothesis is the trans-differentiation of CD117 ${ }^{+}$ILCs in response to the local environment from LN. It has been reported that not only murine ILCs but also human blood and nasal polyp derived ILC2s could trans-differentiate by stimulation of a set of cytokines (IL-1 $\beta$, IL-13, and TGF- $\beta$ ) [36]. However, the majority of $\mathrm{CD} 117^{+}$ILCs shown in our data were not fully functional, cytokine-producing ILC3s (Figs. 1c and 2c). Since the trans-differentiation means the functional or phenotypical change of a differentiated ILC into another subset [37], it would not be appropriate indicating the observed phenomenon of reduced CD117 ${ }^{+}$ILCs in LN as the result of trans-differentiation or plasticity. Overall, it would be appropriate to explain that the reduction of $\mathrm{CD} 117^{+}$ ILCs either in vivo or in vitro is a result of increased differentiation of the ILC progenitors in LN environment.

Given the fact that the IL-1R1 is expressed on CD117 ${ }^{+}$ ILCs, IL-1 $\beta$, which is elevated in LN plasma, was the first candidate factor to induce the differentiation of ILCs. Blocking of IL-1 receptor reversed the phenomenon of reducing percentage of $\mathrm{CD} 117^{+}$ILCs in LN. Considering their marginal effects on the ILC progenitors, however, further exploratory study is still needed to understand the exact mechanism of ILC biology in patients with LN.

To examine the dynamics of ILCs in inflamed LN kidney tissue in real-time, we investigated ILCs in urine. Although the number of ILCs in urine was pretty small, we found that patients with LN had lower numbers of urine $\mathrm{CD} 117^{+}$ILCs than those with other kidney diseases, including AAV and IgAN which also showed reduction of 
those cells in blood as in LN. The finding that only LN ILCs showed a significant correlation with various markers of nephritis might be due to marked changes in the cell numbers in the target organ. The use of urine immune cells as a surrogate for those in renal tissue was thoroughly verified by the recent study by Arazi et al [38]. Immunofluorescence staining of intra-renal CD $117^{+}$ILCs confirmed their presence in a healthy kidney; however, they were barely detected in LN. It is still difficult to verify whether the reduced number of CD $117^{+}$ILCs in LN urine is due to a reduction in the number of ILC progenitors.

Finally, we compared the subset distribution and function of ILCs from patients with active and stable LN. We noted that the reduction in ILC numbers in those with active LN recovered when the disease stabilized. Furthermore, we assessed the correlation between ILCs and various clinical parameters. Changes of eGFR, WBC count [23], and proteinuria amount, all of which reflect LN activity, correlated significantly with a reduction in the total number of ILCs and in the number of CD117 ${ }^{+}$ILCs. In addition, we found that as the number of exhausted ILCs increased, disease status worsens. Reduced cytokine production by ILCs was not limited to a particular subset. This phenomenon could be explained in several ways. First, the reduction in cytokine-producing ILCs could be linked directly to increased differentiation of CD117 ${ }^{+}$ILC progenitors. Decreasing numbers of ILC progenitors could affect the differentiation machinery and contribute to increased ILC dysfunction. Second, the phenomenon we found might be due to exhaustion of ILCs. Compared with $\mathrm{T}$ cells, few studies have reported exhaustion of ILCs [39, 40]. All of these studies were based on murine models, but all linked dysfunction of ILCs to expression of PD-1, a well-known immune inhibitory molecule [41, 42]. Indeed, circulating CD4 $\mathrm{T}$ cells from patients with rheumatoid arthritis show higher expression of PD-1 than those from HC [43]. We also found that expression of PD-1 by ILCs from patients with active LN was higher than that by ILCs from those with stable LN. ILCs from those with active LN produce less cytokine (especially, IL-13 and IL-17A) than those from patients with stable LN. Third, it is possible that other cytokines (not examined here) might be involved in the pathogenesis of LN. In addition to common cytokines such as IFN- $\gamma$, IL-13, and IL-17A, ILCs could secrete amphiregulin [44], GM-CSF [45], and IL-9 [46], all of which have pathogenic or protective effects under various disease conditions.

\section{Conclusions}

In summary, we show here that LN patients harbor reduced numbers of total ILCs and CD $117^{+}$ILCs, and that ILCs show impaired cytokine production. The takehome points are as follows: (1) this is the first study of human ILCs to compare LN with other renal diseases,
(2) urine ILCs were used as a surrogate marker for ILCs in kidney tissues, and (3) the dynamics of ILCs in those with active and stable LN was supported by in vitro experiments with ILC progenitors. A limitation of this study is that there was restricted opportunity to look into the role of ILCs in other autoimmune kidney diseases except SLE, although they also showed similar trends in circulating ILCs. This is, however, the first step to understand the role of ILCs in LN and other autoimmune kidney diseases. Considering their protective role in murine kidney, follow-up studies focusing on the role of ILCs in the pre-clinical status of these diseases when ILCs are abundant and fully functional will increase our understanding of immune reactions in LN.

\section{Supplementary information}

Supplementary information accompanies this paper at https://doi.org/10. 1186/s13075-020-2114-5.

Additional file 1: Figure S1. Phenotype and functional aspects of kidney ILCs from pathologically normal renal tissue obtained from a patient with renal cell carcinoma.

Additional file 2: Figure S2. Immunofluorescence staining of CD $117^{+}$ ILCS (c, d; found as CD3 ${ }^{-}$CD117 $\left.{ }^{+}\right)$in renal tissue showed that they were decreased in renal tissue of (b) LN, compared to that of (a) HC. (c) DAPI (blue), CD3 (green), and CD117 (red), and merged image. (d) The number of $\mathrm{CD}^{-} \mathrm{CD} 117^{+}$cells within the same area in renal tissue of $\mathrm{HC}$ and $\mathrm{LN}$. Pink arrow: $\mathrm{CD}^{+} \mathrm{T}$ cells, white arrow: $\mathrm{CD} 117^{+} \mathrm{ILCS}$, orange arrow: $\mathrm{CD}^{+}$ CD $117^{+} T$ cells. ${ }^{*} P<0.05$ (Student's t test).

Additional file 3: Figure S3. Correlation between the percentage of urine ILCs ((a) CD $117^{+} \mathrm{ILCs}$ and (b) CRTH2 ${ }^{+} \mathrm{ILCS}$ ) and clinical parameters including serum BUN and creatinine, activity and chronicity index. ${ }^{*} P<$ 0.05 (Pearson correlation).

Additional file 4: Figure S4. Blood ILCS from a healthy control before in vitro culture, related to Fig. 5a, b.

Additional file 5: Figure S5. PD-1 expressing ILCs within PBMCS did not express IFN- $\gamma$, IL-13, or IL-17A. ILCs in both (a) HC and (b) LN.

Additional file 6: Figure S6. Working model. In healthy kidney, cytokine producing ILCs maintain tissue homeostasis. In patients with LN, reduced numbers of ILC progenitor cells result in exhaustion of the ILCS with increased expression of PD-1. A reduction of functional ILCs might associate with increased severity of LN. Figure contains some images adapted from SMART (Servier Medical Art; http://smart.servier.com/), licensed under a Creative Common Attribution 3.0 Generic License.

\section{Abbreviations}

AAV: ANCA-associated vasculitis; BUN: Blood urea nitrogen; C3: Complement component 3; C4: Complement component 4; CRP: C-reactive protein; dsDNA: Double-stranded DNA; FSGS: Focal segmental glomerulosclerosis; HC: Healthy control; IgAN: IgA nephropathy; ILC: Innate lymphoid cell; LN: Lupus nephritis; MCD: Minimal change disease; PBMC: Peripheral blood mononuclear cell; UPCR: Urine protein to creatinine ratio; WBC: White blood cell

\section{Acknowledgements}

The bio-specimens for the study were provided by the Seoul National University Human Biobank, a member of the National Biobank of Korea, which is supported by the Ministry of Health and Welfare, Korea.

\section{Authors' contributions}

SR designed the study, performed the experiments, analyzed the data, and wrote the manuscript. EYL, DKK, and YSK recruited the study subjects, analyzed clinical data, and wrote the manuscript. DHC and JHK contributed 
to data analysis and manuscript preparation. $\mathrm{HL}$ designed the study, supervised sample acquisition, and revised the manuscript. HYK designed the study and wrote the manuscript. All authors read and approved the final manuscript.

\section{Funding}

This study was supported by a grant from the Korea Healthcare Technology R\&D Project, Ministry of Health and Welfare, Korea (H115C3083 and HI15C1736), and a grant from the National Research Foundation of Korea (SRC 2017R1A5A1014560).

\section{Availability of data and materials}

All data generated or analyzed during this study are included in this published article.

\section{Ethics approval and consent to participate}

All subjects enrolled in this study provided written informed consent. The study protocol was approved by the Seoul National University Hospital Institutional Review Board (IRB number 1709-105-887).

\section{Consent for publication}

Not applicable

\section{Competing interests}

The authors declare that they have no competing interests.

\begin{abstract}
Author details
'Laboratory of Mucosal Immunology, Department of Biomedical Sciences, Seoul National University College of Medicine, Seoul 03080, South Korea. ${ }^{2}$ Division of Rheumatology, Department of Internal Medicine, Seoul National University Hospital, Seoul, South Korea. ${ }^{3}$ Division of Nephrology, Department of Internal Medicine, Seoul National University Hospital, Seoul, South Korea. ${ }^{4}$ Kidney Research Institute, Seoul National University College of Medicine, Seoul 03080, South Korea. ${ }^{5}$ Department of Pathology, Seoul National University College of Medicine, Seoul, South Korea. 'Laboratory of Immune Regulation, Department of Biomedical Sciences, Seoul National University College of Medicine, Seoul, South Korea. ${ }^{7}$ College of Life Sciences and Biotechnology, Korea University, Seoul, South Korea. ${ }^{8}$ Institute of Allergy and Clinical Immunology, Seoul National University Medical Research Center, Seoul, South Korea.
\end{abstract}

\section{Received: 19 July 2019 Accepted: 4 February 2020} Published online: 29 March 2020

\section{References}

1. Tsokos GC. Systemic lupus erythematosus. N Engl J Med. 2011;365(22):2110-21.

2. Anders HJ, Rovin B. A pathophysiology-based approach to the diagnosis and treatment of lupus nephritis. Kidney Int. 2016;90(3):493-501.

3. Deng Y, Tsao BP. Genetic susceptibility to systemic lupus erythematosus in the genomic era. Nat Rev Rheumatol. 2010;6(12):683-92.

4. Hahn BH. Antibodies to DNA. N Engl J Med. 1998;338(19):1359-68.

5. Almaani S, Meara A, Rovin BH. Update on lupus nephritis. Clin J Am Soc Nephrol. 2017;12(5):825-35

6. Doria A, Cervera R, Gatto M, Chehab G, Schneider M. The new targeted therapy in systemic lupus erythematosus: is the glass half-full or half-empty? Autoimmun Rev. 2017;16(11):1119-24.

7. Sciascia S, Radin M, Yazdany J, Levy RA, Roccatello D, Dall'Era M, Cuadrado MJ. Efficacy of belimumab on renal outcomes in patients with systemic lupus erythematosus: a systematic review. Autoimmun Rev. 2017:16(3):287-93.

8. Eberl G, Di Santo JP, Vivier E. The brave new world of innate lymphoid cells. Nat Immunol. 2015;16(1):1-5.

9. Lim Al, Li Y, Lopez-Lastra S, Stadhouders R, Paul F, Casrouge A, Serafini N, Puel A, Bustamante J, Surace L, et al. Systemic human ILC precursors provide a substrate for tissue ILC differentiation. Cell. 2017;168(6):1086-100 e1010.

10. Bar-Ephraim YE, Koning JJ, Burniol Ruiz E, Konijn T, Mourits VP, Lakeman KA, Boon L, Bogels M, van Maanen JP, Den Haan JMM, et al. CD62L is a functional and phenotypic marker for circulating innate lymphoid cell precursors. J Immunol. 2019;202(1):171-82.

11. Wenink MH, Leijten EFA, Cupedo T, Radstake T. Review: innate lymphoid cells: sparking inflammatory rheumatic disease? Arthritis Rheumatol. 2017;69(5):885-97.
12. Shikhagaie MM, Germar K, Bal SM, Ros XR, Spits H. Innate lymphoid cells in autoimmunity: emerging regulators in rheumatic diseases. Nat Rev Rheumatol. 2017;13(3):164-73.

13. Wohlfahrt T, Usherenko S, Englbrecht M, Dees C, Weber S, Beyer C, Gelse K, Distler O, Schett G, Distler JH, et al. Type 2 innate lymphoid cell counts are increased in patients with systemic sclerosis and correlate with the extent of fibrosis. Ann Rheum Dis. 2016;75(3):623-6.

14. Ciccia F, Guggino G, Rizzo A, Saieva L, Peralta S, Giardina A, Cannizzaro A, Sireci G, De Leo G, Alessandro R, et al. Type 3 innate lymphoid cells producing $\mathrm{IL}-17$ and $\mathrm{LL}-22$ are expanded in the gut, in the peripheral blood, synovial fluid and bone marrow of patients with ankylosing spondylitis. Ann Rheum Dis. 2015;74(9):1739-47

15. Riedel JH, Becker M, Kopp K, Duster M, Brix SR, Meyer-Schwesinger C, Kluth LA, Gnirck AC, Attar M, Krohn S, et al. IL-33-mediated expansion of type 2 innate lymphoid cells protects from progressive glomerulosclerosis. J Am Soc Nephrol. 2017;28(7):2068-80.

16. Cameron GJM, Cautivo KM, Loering S, Jiang SH, Deshpande AV, Foster PS, McKenzie ANJ, Molofsky AB, Hansbro PM, Starkey MR. Group 2 innate lymphoid cells are redundant in experimental renal ischemia-reperfusion injury. Front Immunol. 2019;10:826.

17. Cao Q, Wang Y, Niu Z, Wang C, Wang R, Zhang Z, Chen T, Wang XM, Li Q, Lee WW, et al. Potentiating tissue-resident type 2 innate lymphoid cells by IL-33 to prevent renal ischemia-reperfusion injury. J Am Soc Nephrol. 2018:29(3):961-76

18. Huang Q, Niu Z, Tan J, Yang J, Liu Y, Ma H, Lee W, Sun S, Song X, Guo M, et al. IL-25 elicits innate lymphoid cells and multipotent progenitor type 2 cells that reduce renal ischemic/reperfusion injury. J Am Soc Nephrol. 2015;26(9):2199-211.

19. Duster M, Becker M, Gnirck AC, Wunderlich M, Panzer U, Turner JE. T cell-derived IFN-gamma downregulates protective group 2 innate lymphoid cells in murine lupus erythematosus. Eur J Immunol. 2018; 48(8):1364-75.

20. Hochberg MC. Updating the American College of Rheumatology revised criteria for the classification of systemic lupus erythematosus. Arthritis Rheum. 1997:40(9):1725.

21. Petri M, Orbai AM, Alarcon GS, Gordon C, Merrill JT, Fortin PR, Bruce IN, Isenberg D, Wallace DJ, Nived O, et al. Derivation and validation of the Systemic Lupus International Collaborating Clinics classification criteria for systemic lupus erythematosus. Arthritis Rheum. 2012;64(8):2677-86.

22. Mallone R, Mannering SI, Brooks-Worrell BM, Durinovic-Bello I, Cilio CM, Wong FS, Schloot NC, T-Cell Workshop Committee loDS. Isolation and preservation of peripheral blood mononuclear cells for analysis of islet antigen-reactive T cell responses: position statement of the T-Cell Workshop Committee of the Immunology of Diabetes Society. Clin Exp Immunol. 2011;163(1):33-49.

23. Fayyaz A, Igoe A, Kurien BT, Danda D, James JA, Stafford HA, Scofield RH. Haematological manifestations of lupus. Lupus Sci Med. 2015;2(1):e000078.

24. Chen L, Youssef $Y$, Robinson C, Ernst GF, Carson MY, Young KA, Scoville SD, Zhang $X$, Harris R, Sekhri P, et al. CD56 expression marks human group 2 innate lymphoid cell divergence from a shared NK cell and group 3 innate lymphoid cell developmental pathway. Immunity. 2018; 49(3):464-76 e464.

25. Smith MA, Henault J, Karnell JL, Parker ML, Riggs JM, Sinibaldi D, Taylor DK, Ettinger R, Grant EP, Sanjuan MA, et al. SLE plasma profiling identifies unique signatures of lupus nephritis and discoid lupus. Sci Rep. 2019;9(1):14433.

26. Vivier E, Artis D, Colonna M, Diefenbach A, Di Santo JP, Eberl G, Koyasu S, Locksley RM, McKenzie ANJ, Mebius RE, et al. Innate lymphoid cells: 10 years on. Cell. 2018;174(5):1054-66.

27. Scoville SD, Mundy-Bosse BL, Zhang MH, Chen L, Zhang X, Keller KA, Hughes T, Chen L, Cheng S, Bergin SM, et al. A progenitor cell expressing transcription factor RORgammat generates all human innate lymphoid cell subsets. Immunity. 2016:44(5):1140-50.

28. Guo C, Zhou M, Zhao S, Huang Y, Wang S, Fu R, Li M, Zhang T, Gaskin F, Yang $N$, et al. Innate lymphoid cell disturbance with increase in ILC1 in systemic lupus erythematosus. Clin Immunol. 2019;202:49-58.

29. Kruize AA, Leijten EFA, van den Hoogen LL, Hartgring SAY, Blokland SLM, Radstake TRDJ, van Roon JAG, Fritsch R. Increased expression of Fas on group 2 and 3 innate lymphoid cells is associated with an interferon signature in systemic lupus erythematosus and Sjögren's syndrome. Rheumatology (Oxford). 2019;58(10):1740-5.

30. Kloverpris HN, Kazer SW, Mjosberg J, Mabuka JM, Wellmann A, Ndhlovu Z, Yadon MC, Nhamoyebonde S, Muenchhoff M, Simoni Y, et al. Innate 
lymphoid cells are depleted irreversibly during acute HIV-1 infection in the absence of viral suppression. Immunity. 2016;44(2):391-405.

31. Zhang Z, Cheng L, Zhao J, Li G, Zhang L, Chen W, Nie W, Reszka-Blanco NJ, Wang FS, Su L. Plasmacytoid dendritic cells promote HIV-1-induced group 3 innate lymphoid cell depletion. J Clin Invest. 2015;125(9):3692-703.

32. Mudd JC, Busman-Sahay K, DiNapoli SR, Lai S, Sheik V, Lisco A, Deleage C, Richardson B, Palesch DJ, Paiardini M, et al. Hallmarks of primate lentiviral immunodeficiency infection recapitulate loss of innate lymphoid cells. Nat Commun. 2018;9(1):3967.

33. Xu H, Wang X, Lackner AA, Veazey RS. Type 3 innate lymphoid cell depletion is mediated by TLRs in lymphoid tissues of simian immunodeficiency virus-infected macaques. FASEB J. 2015;29(12):5072-80.

34. Nagasawa M, Heesters BA, Kradolfer CMA, Krabbendam L, Martinez-Gonzalez I, de Bruijn MJW, Golebski K, Hendriks RW, Stadhouders R, Spits H, et al. KLRG1 and NKp46 discriminate subpopulations of human CD117(+ )CRTH2(-) ILCs biased toward ILC2 or ILC3. J Exp Med. 2019;216(8):1762-76.

35. Crow MK. Type I interferon in the pathogenesis of lupus. J Immunol. 2014; 192(12):5459-68.

36. Golebski K, Ros XR, Nagasawa M, van Tol S, Heesters BA, Aglmous H, Kradolfer CMA, Shikhagaie MM, Seys S, Hellings PW, et al. IL-1beta, IL-23, and TGF-beta drive plasticity of human ILC2s towards IL-17-producing ILCS in nasal inflammation. Nat Commun. 2019:10(1):2162.

37. Wagner M, Moro K, Koyasu S. Plastic heterogeneity of innate lymphoid cells in cancer. Trends Cancer. 2017;3(5):326-35.

38. Arazi A, Rao DA, Berthier CC, Davidson A, Liu Y, Hoover PJ, Chicoine A, Eisenhaure TM, Jonsson AH, Li S, et al. The immune cell landscape in kidneys of patients with lupus nephritis. Nat Immunol. 2019;20(7):902-14.

39. Taylor S, Huang Y, Mallett G, Stathopoulou C, Felizardo TC, Sun MA, Martin EL, Zhu N, Woodward EL, Elias MS, et al. PD-1 regulates KLRG1(+) group 2 innate lymphoid cells. J Exp Med. 2017;214(6):1663-78.

40. Oldenhove G, Boucquey E, Taquin A, Acolty V, Bonetti L, Ryffel B, Le Bert M, Englebert K, Boon L, Moser M. PD-1 is involved in the Dysregulation of type 2 innate lymphoid cells in a murine model of obesity. Cell Rep. 2018;25(8): 2053-60 e2054.

41. Hashimoto M, Kamphorst AO, Im SJ, Kissick HT, Pillai RN, Ramalingam SS, Araki K, Ahmed R. CD8 T cell exhaustion in chronic infection and cancer: opportunities for interventions. Annu Rev Med. 2018:69:301-18.

42. Thommen DS, Schumacher TN. T cell dysfunction in cancer. Cancer Cell. 2018:33(4):547-62

43. Frenz T, Grabski E, Buschjager D, Vaas LA, Burgdorf N, Schmidt RE, Witte T, Kalinke U. CD4(+) T cells in patients with chronic inflammatory rheumatic disorders show distinct levels of exhaustion. J Allergy Clin Immunol. 2016; 138(2):586-9 e510.

44. Jeffery HC, McDowell P, Lutz P, Wawman RE, Roberts S, Bagnall C, Birtwistle J, Adams DH, Oo YH. Human intrahepatic ILC2 are IL-13positive amphiregulinpositive and their frequency correlates with model of end stage liver disease score. PLoS One. 2017;12(12):e0188649.

45. Hirota $\mathrm{K}$, Hashimoto M, Ito Y, Matsuura M, Ito H, Tanaka M, Watanabe H, Kondoh G, Tanaka A, Yasuda K, et al. Autoimmune Th17 cells induced synovial stromal and innate lymphoid cell secretion of the cytokine GM-CSF to initiate and augment autoimmune arthritis. Immunity. 2018;48(6):1220-32 e1225.

46. Rauber S, Luber M, Weber S, Maul L, Soare A, Wohlfahrt T, Lin NY, Dietel K, Bozec A, Herrmann M, et al. Resolution of inflammation by interleukin-9producing type 2 innate lymphoid cells. Nat Med. 2017;23(8):938-44.

\section{Publisher's Note}

Springer Nature remains neutral with regard to jurisdictional claims in published maps and institutional affiliations.

Ready to submit your research? Choose BMC and benefit from:

- fast, convenient online submission

- thorough peer review by experienced researchers in your field

- rapid publication on acceptance

- support for research data, including large and complex data types

- gold Open Access which fosters wider collaboration and increased citations

- maximum visibility for your research: over $100 \mathrm{M}$ website views per year

At BMC, research is always in progress.

Learn more biomedcentral.com/submissions 